\title{
Cloning, analysis and one-step disruption of the ARG5,6 gene of Candida albicans
}

\author{
A. Negredo, L. Monteoliva, C. Gil, J. Pla and C. Nombela \\ Author for correspondence: J. Pla. Tel: +34 1394 1617. Fax: +34 13941745. \\ e-mail: jesuspla@eucmax.sim.ucm.es
}

Departamento de Microbiología II, Facultad de Farmacia, Universidad Complutense de Madrid, Avda. Ramón y Cajal s/n, 28040 Madrid, Spain
The ARG5,6 gene from the dimorphic fungus Candida albicans was cloned by functional complementation of the arginine auxotrophy present in strain EL2 ( Arg $^{-}$) using a gene library constructed in the double autonomously replicating sequence vector pRM1. Sequence analysis revealed a putative 857 amino acid polypeptide (95 kDa) which showed high homology (63\% protein identity) to the Saccharomyces cerevisiae ARG5,6 gene. Similarly to the $S$. cerevisiae gene, the C. albicans ARG5,6 gene is responsible for both the acetylglutamate kinase and acetylglutamyl-phosphate reductase activities, the second and third steps of arginine biosynthesis at the mitochondria. The C. albicans ARG5,6 gene complemented the arg6 mutation present in S. cerevisiae (strain D160-4D) on a yeast episomal plasmid using its own regulatory signals. A set of nonintegrative high-efficiency plasmid vectors based on this gene marker was constructed and a null $C$. albicans arg5,6 $\Delta$ strain was obtained using the common URA3-blaster strategy. In addition, we generated an arg5,6 $\Delta$ null mutant in a single transformation event, thus improving the basic strategy for generating gene deletions in C. albicans.

Keywords: ARG5,6, Candida albicans, molecular biology, gene disruption, arginine

\section{INTRODUCTION}

Despite the importance of Candida albicans as a model pathogenic yeast, obtaining information about its genetics has been difficult due to its diploidy and lack of sexual cycle (Kurtz et al., 1990; Scherer \& Magee, 1990). Although C. albicans genetics have largely relied on Saccharomyces cerevisiae as an intermediate genetic host, some of the most interesting and peculiar processes in C. albicans, such as its ability to switch between a yeast and a mycelial form of growth and its pathogenicity to humans (Odds, 1988), cannot readily be studied vicariously through a knowledge of $S$. cerevisiae. The development of an efficient transformation system, with the contruction of both autoreplicative plasmids and suitable host strains, is then one of the major goals to facilitate genetic analysis of the biology and pathogenicity of C. albicans.

Although Kurtz and colleagues described both the integrative transformation (Kurtz et al., 1986) and the

The EMBL accession number for the nucleotide sequence reported in this paper is $X 98880$. development of autoreplicative plasmids (Kurtz et al., 1987) in Candida, plasmids often multimerize in C. albicans leading to difficulties in their isolation (Goshorn et al., 1992). We recently developed plasmids carrying two independent autonomously replicating sequences (Cannon et al., 1990; Herreros et al., 1992) that allow the introduction and recovery of DNA in C. albicans (Pla et al., 1995). A second important tool in C. albicans molecular genetics is the availability of isogenic auxotrophic host strains. Although both UV-enhanced mitotic recombination (Kelly et al., 1987; Sadhu et al., 1992) and toxic metabolites (Gorman et al., 1991) have been used for gene disruption in C. albicans, the most commonly employed system now used makes use of the strategy adapted by Fonzi \& Irwin (1993), which requires two sequential steps of gene disruption using the C. albicans URA3 gene flanked by a heterologous bisG sequence from Salmonella typhimurium. This process could, however, be shortened if different genetic markers and appropriate strains were available for sequential disruptions. Although some nutritional genes have been isolated (Rosenbluh et al., 1985; Goshorn et al., 1992; Hoyer et al., 1994; Pla et al., 1995), there are no reports of strains with more than one auxotrophic marker obtained entirely by gene disruption. 
In this work, we describe the isolation of the ARG5,6 gene from $C$. albicans by complementation of a mutagenized $C$. albicans arginine auxotrophic strain and its use in developing a genetic transformation system. In addition, we have constructed a triple ura3 $\Delta$ bis $1 \Delta \arg 5,6 \Delta$ strain and demonstrate the generation of a homozygous arg5,6 null strain in a single transformation event.

\section{METHODS}

Strains and growth conditions. C. albicans and Escherichia coli strains are listed in Table 1. C. albicans strain 1001 (ATCC 64385), a wild-type strain from the Spanish Type Culture Collection, was used as the source of genomic DNA for the construction of a genomic library in plasmid pRM1 (Pla et al., 1995). The following S. cerevisiae strains, obtained from the Yeast Genetic Stock Center (Berkeley, CA, USA), were used to check the function of the cloned C. albicans ARG5,6 gene by complementation: X3163-4C (MATa arg1 met1 trp3 ade5 ura3 leu1 mal gal2); STX63-8B (MATa arg2 lys4 lys11 trp4 ade2 tyr1 rad2 gal2); STX9-1A (MATa arg3 ade2 gal2); $\mathrm{AB} 18-20 \mathrm{~A}$ (MAT a arg4-8 ade2 ade 5 CUP1 ${ }^{\mathrm{r}}$ leu2-3, 112 ura3-52 met13 cyh ${ }^{\mathrm{r}}$ ); D160-4D (MATa arg6 ura3 hom3 bis1 trp2 ade1 met1 gal2); X1049-9C (MATa arg8 trp1 ura3 his3 asp5); XJB17-2 (MATa arg9 thr4 leu2 ade6 gal2); and STX14-1C (MATa arg10 ade2 gal2). Yeast strains were grown in either YED medium $(1 \%, \mathrm{w} / \mathrm{v}$, yeast extract; $2 \%, \mathrm{w} / \mathrm{v}$, glucose) or SD minimal medium ( $0.67 \%$ Yeast Nitrogen Base without amino acids; $2 \%, \mathrm{w} / \mathrm{v}$, glucose), supplemented with uridine, histidine or arginine at $25 \mathrm{\mu g} \mathrm{m}^{-1}$, depending on the nutritional requirements, with continuous shaking at $30^{\circ} \mathrm{C}$. C. albicans $\mathrm{Ura}^{-}$revertants were selected on 5 -fluoroorotic acid plates as described previously by Navarro-García $e t$ al. (1995). E. coli strains were grown in Luria-Bertani (LB) or Terrific Broth (TB) at $37^{\circ} \mathrm{C}$ supplemented with $100 \mu \mathrm{g}$ ampicillin $\mathrm{ml}^{-1}$ for plasmid selection.
DNA manipulations. All DNA manipulations were carried out following standard procedures (Sambrook et al., 1989). Southern hybridization analysis was carried out using the Nonradioactive Labelling and Detection kit (Boehringer Mannheim) under high-stringency conditions on positively charged nylon membranes. For the determination of the ARG5,6 sequence, a $3.8 \mathrm{~kb} B a m \mathrm{HI}-B a m \mathrm{HI}$ insert from plasmid pAN1 was purified from agarose gels and sonicated to generate random fragments $(300-600 \mathrm{bp}$ in size) which were subcloned into the SmaI site of pUC19. Plasmid DNA was purified from E. coli transformants with Qiagen (Diagen) and both strands were sequenced with an Automated Sequencer (ALF; Pharmacia) according to Sanger et al. (1977) using the Universal and Reversal fluoresceinated primers. Sequence comparisons and homologies were carried out using the FASTA algorithm (Pearson \& Lipman, 1988). DNA sequence analysis was performed with the PCGENE software.

Plasmid constructions and gene disruption. Plasmid pRM1, an efficient non-integrative plasmid, as well as its derived gene library have been described before (Pla et al., 1995). The $E$. coli/yeast shuttle vector YEp352 has also been described previously by Hill et al. (1986). YEpARG was obtained by subcloning a $3.8 \mathrm{~kb}$ Bam $\mathrm{HI}-$ Bam HI fragment from pAN1 into the BamHI site of YEp352. Plasmid pAN8, an autoreplicative plasmid which transforms $C$. albicans with high efficiency $\left[>5 \times 10^{3}\right.$ transformants $\left.(\mu \mathrm{g} \mathrm{DNA})^{-1}\right]$ and which is representative of a set of autoreplicative plasmids bearing this marker, was obtained by replacing the NaeI-SmaI fragment of pRM10 (Pla et al., 1995) with a blunt-ended BamHI-BamHI fragment from pAN1. For the disruption of the C. albicans ARG5,6 gene, the same $4 \mathrm{~kb} \mathrm{BamHI-BamHI}$ fragment was subcloned into pUC19 yielding pUC-ARG. An internal SnaBI-BglII fragment (which comprised most of the ARG6 domain) was then replaced with either the BamHI-BglII fragment of pCUB-6 (Fonzi \& Irwin, 1993) (carrying the hisG-URA3-his G cassette) or the KpnI-SspI fragment from YEp-HISX (Pla et al., 1995) (carrying the C. albicans HIS1

\section{Table 1. Strains used in this work}

\begin{tabular}{|c|c|c|c|}
\hline Organism & Strain & Genotype/phenotype & Origin \\
\hline C. albicans & $1001 *$ & Wild-type & Gil et al. (1988) \\
\hline C. albicans & SC5314 & Wild-type & Gillum et al. (1984) \\
\hline C. albicans & EL2† & $\mathrm{Arg}^{-} \mathrm{Ade}^{-}$ & Gil et al. (1988) \\
\hline C. albicans & CAI4 & ura $3 \Delta::$ imm $434 /$ ura $3 \Delta:: i m m 434$ & Fonzi \& Irwin (1993) \\
\hline C. albicans & 1006 & MPA1 ser 57 ura 3 lys 1 arg $4 \ddagger$ & \\
\hline C. albicans & RM1000 & ura $3 \Delta::$ imm $434 /$ ura $3 \Delta:: i m m 434$ his $1 \Delta::$ his $G$ bis $1 \Delta::$ his $G$ & Unpublished \\
\hline C. albicans & $\mathrm{CNC40}$ & 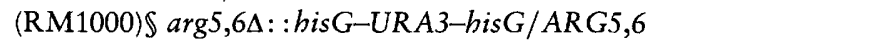 & This work \\
\hline C. albicans & CNC41 & $(\mathrm{RM} 1000) \S \arg 5,6 \Delta:$ : his $G / A R G 5,6$ & This work \\
\hline C. albicans & CNC42 & 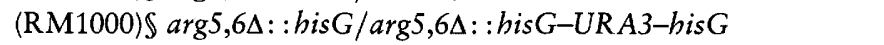 & This work \\
\hline C. albicans & CNC43 & $(\mathrm{RM} 1000) \$ \arg 5,6 \Delta::$ hisG $/ \arg 5,6 \Delta::$ his $G$ & This work \\
\hline C. albicans & CNC44 & 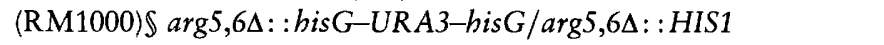 & This work \\
\hline E. coli & MC1061 & araD139 $\Delta($ ara-leu $) 7697 \Delta$ (lac) X74 galu galK straA & Navarro-García et al. (1995) \\
\hline E. coli & $\mathrm{DH} 5 \alpha \mathrm{F}^{\prime}$ & $\begin{array}{l}\text { K12 } \Delta(\text { lacZYA-argF }) \mathrm{U} 169 \text { supE44 thi1 recA1 endA1 hsdR17 gyrA } \\
\text { relA1 ( } \phi 80 \text { lacZ } \triangle \mathrm{M} 15) \mathrm{F}^{\prime}\end{array}$ & Hanahan (1988) \\
\hline
\end{tabular}

*A wild-type strain from the Colección Española de Cultivos Tipo (Spanish Type Culture Collection), ATCC 64385.

+ An $\mathrm{Arg}^{-} \mathrm{Ade}^{-}$auxotroph derived from strain 1001.

‡Designated arg57 (Goshorn \& Scherer, 1989) and later shown to be arg4 mutant (Hoyer et al., 1994).

§RM1000 background. 
gene) yielding, respectively, pUC-ARG-U and pUC-ARG-H. Homologous integration of these constructs was achieved by transformation using DNA that had been linearized with $S c a$ I and BamHI. The genotype of all strains was checked by Southern analysis using a ClaI-ScaI fragment of C. albicans $A R G 5,6$ as a probe and digesting the genomic DNA of the transformants with HpaI-ClaI (Fig. 1).

Genetic transformation procedures. C. albicans was transformed using the standard protoplast method with minor modifications (Herreros et al., 1992; Pla et al., 1995). Gene disruption of $C$. albicans $A R G 5,6$ by cotransformation of pUC-ARG-U and pUC-ARG-H was optimized with respect to the concentration of both DNAs used in the assay. Protoplast fusions were performed as described previously (Gil et al., 1988). Electroporation was used for the recovery of plasmid DNA from C. albicans transformants (Pla et al., 1995).

\section{RESULTS AND DISCUSSION}

\section{Isolation of the C. albicans ARG5,6 gene}

Strain EL2 is an arginine and adenine auxotroph obtained through ethylmethane sulfonate mutagenesis of strain 1001 as described previously (Gil et al., 1990). This auxotrophic mutant was used for the generation of a set of $C$. albicans mutants altered in their ability to undergo the dimorphic transition (Gil et al., 1988, 1990). We first verified that the arginine biosynthetic defect present in strains EL2 and 1006 (arg4; Hoyer et al., 1994) was different since it was possible to isolate prototrophic

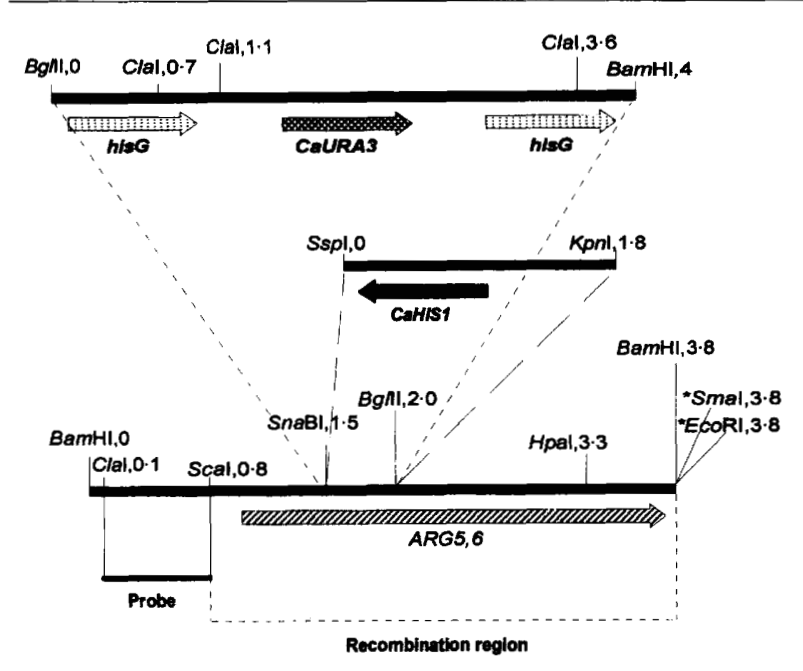

$0.50 \mathrm{~kb}$

Fig. 1. Physical map of the ARG5,6 locus and its disruption strategy. The hisG-URA3-hisG cassette and the HIS1 gene were used to replace an internal fragment of the C. albicans ARG5,6 gene. Only the relevant restriction sites are shown, with numbers indicating the position in $\mathrm{kb}$. The asterisks indicate restriction sites that are derived from the multiple cloning site of the pUC polylinker and are not found in the genomic DNA. Arrows represent the structural genes. The probe used in Southern biot analysis and the recombination region are indicated (see Methods). hybrids when parasexual crosses between both strains were carried out. A gene library constructed in the double autonomously replicating sequence vector pRM1 (Pla et al., 1995) was used to isolate the complementing gene. Screening of approximately 50000 transformants yielded 14 prototrophs after $7 \mathrm{~d}$ growth in selective medium. Only one of them was characterized further. Plasmid DNA, designated pAN1, was extracted from this transformant and was shown to be capable of complementing the auxotrophic phenotype upon retransformation. Standard restriction analysis revealed an insert of approximately $7 \mathrm{~kb}$, with a minimal $3.8 \mathrm{~kb}$ Bam HI-Bam HI complementing region (Fig. 1). When present in the episomal plasmid YEp352 (plasmid YEpARG), this DNA was shown to complement the arg6 defect present in strain D160-4D (an arg6 mutant). These results demonstrate the usefulness of these replicative plasmids (Cannon et al., 1992; Pla et al., 1995) in C. albicans genetic manipulation.

\section{Sequence analysis of the C. albicans ARG5,6 gene}

Sequence analysis of the insert revealed a putative ORF of 857 amino acids $(95 \mathrm{kDa})$. This protein was $63 \%$ identical to the predicted translation product of the ARG5,6 gene of S. cerevisiae (54\% identical to Schizosaccharomyces pombe Arg 5,6p), thus indicating that we had cloned the C. albicans ARG5,6 gene. In S. cerevisiae, this gene is responsible for the generation of a polypeptide which is post-translationally processed yielding two mature polypeptides that encode the acetylglutamate kinase (ARG6) and acetylglutamate-phosphate reductase (ARG5) activities in the arginine biosynthetic pathway (Boonchird et al., 1991). Thus, in C. albicans, the genetic organization of the $A R G 5,6$ locus is apparently similar to that in S. cerevisiae (Boonchird et al., 1991) and Schiz. pombe (van Huffel et al., 1992) and contrasts with the situation in prokaryotes where each activity is encoded by a separate gene. A putative TATA box was found around position 253 (Fig. 2), while a putative transcription termination signal was at 2943-2967 (based on their homology to S. cerevisiae consensus signals). No obvious $S$. cerevisiae GCN boxes (consensus sequence TGACTC) could be found in the 5 -upstream region, as has been observed for the $C$. albicans ARO3 (Pereira \& Livi, 1995) and ARG4 (Hoyer et al., 1994) genes. In addition to the arg4 mutant strains 1006 and TMSU221, another complementation group designated $\arg 100$ has been described for strains A642, hOG318, hOG357, FC18-6 and WC-5-4 (Hoyer et al., 1994) located on the R chromosome. pAN8, an autoreplicative plasmid bearing the $A R G 5,6$ gene, did not complement hOG318 arginine auxotrophy.

\section{Construction of an arg5,6 $\Delta$ null mutant}

We used two different strategies to obtain an arg5,6 mutant in the ura3 $\Delta$ bis $1 \Delta$ strain RM1000 background. We first used the URA3-blaster protocol to generate an arg5,6 6 mutant. Using the URA3 marker (construction 


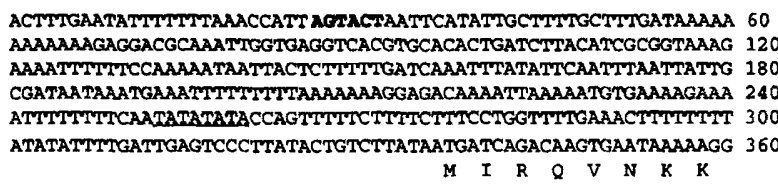

CTATTTCAAACAGTCTTTTCAAAAGATTATCGCTCAGCGGTAGTGCITTIGCTAATATTA 420 $\begin{array}{llllllllllllllllllll}A & I & S & N & S & L & F & K & R & \text { L } & S & \text { L } & S & G & S & A & F & A & N & I\end{array}$

CAGCCAATAAAAAATCGTCCACTCATCAACTCAACCAAAAGACTCAATTAGCAAATGTTC 480

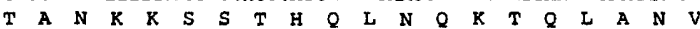
GTTTCTATTCAACAAAATCCACTGTTATTCAGTTATTGAACAACATTGGTTCCAAACGTG 540 $\begin{array}{lllllllllllllllllllll}R & F & Y & S & T & K & S & T & \text { V } & \text { I } & Q & \text { Q } & \text { L } & \text { L } & \text { N } & \text { N } & \text { I } & \text { G } & S & K & R\end{array}$

AAGTTGAACAATACTTGAAATATTTCACTTCGGTATCTCAACAACAATTTGCTGTGATCA 600

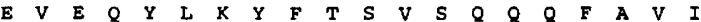

AAGTTGGTGGTGCAATTATCACCCAACAATTGAACGAATTAGCATCATGCTTGGCATITT 660

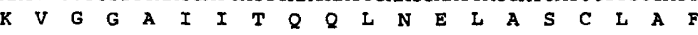

TGTATCATGTIGGTTIGTACCCTATTGTTTTACATGGTACTGGTCCACAAATCAATGAAT 720 $\begin{array}{lllllllllllllllllllll}L & Y & \text { I } & V & G & \text { L } & Y & P & I & V & \text { L } & H & G & T & G & P & Q & I & N & E\end{array}$

TGTTGGAAACGAGGTGTTGAACCAGAATATATTGATGGTATCAGAATCACCAACCCCA 780

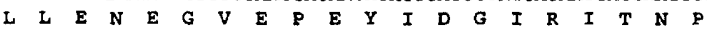

AAACAATGGAAGTAGTACGTAAGTGTTTCTTAGAGCAAAACTTGCGTTTGGTGACAGCTT 840 $\begin{array}{llllllllllllllllllllll}\text { K } & \text { T } & \text { M } & \text { E } & \text { V } & \text { V } & \text { R } & \text { K } & \text { C } & \text { F } & \text { L } & \text { E } & \mathbf{Q} & \text { N } & \text { L } & \text { R } & \text { L } & \text { V } & \text { T } & \text { A }\end{array}$

TGGAAAAATTGGTGTTCACGCTCGTCCAATAACTGCTGGTGTATTTGAAGCTGAATATT 900 $\begin{array}{lllllllllllllllllllll}I & E & K & I & G & V & H & A & R & P & I & T & A & G & V & F & E & A & E & Y\end{array}$

TGGATAAGGACAAGTATCAATTAGTTGGTAAAATCACTAGTGTCAACAAATCCCCAGTTG 960 $\begin{array}{llllllllllllllllllll} & D & K & D & K & Y & Q & L & V & G & K & I & T & S & V & N & K & S & P & V\end{array}$

AAGCTGCCATCAATAGTGGATACTTGCCAATTTTGACATCTTTGGCCGAAACCTCTCTGG 1020 $\begin{array}{llllllllllllllllllll}E & A & A & I & N & S & G & Y & L & P & I & L & T & S & L & A & E & T & S & S\end{array}$

GTCAATPATTAAATGTCAATGCCGACGTTGCTGCCGGAGAATTAGCTCGTGAATTTGAAC 1080

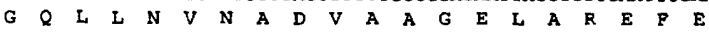

CTITGAAAATTGTTTACTTGAATGAAAAAGTGGTATTATAAACGGAAACACTGGTGAGA 1140

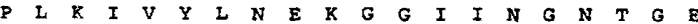

AAGTTTCTGCTATCAATTTGGATGAAGATATGAAGATTTGTTAAAGGAAATTGGGTTA 1200 $\begin{array}{llllllllllllllllllll}X & V & S & A & I & N & L & D & E & E & Y & E & D & L & L & K & E & S & W & V\end{array}$

AATATGGTACTAAATTGAAGATCAAGGAAATTCACGATTTATTACAACATTTGCCAAGAT 1260 $\begin{array}{llllllllllllllllllllll}K & Y & G & T & K & L & K & I & K & E & I & H & D & L & L & Q & H & L & P & R\end{array}$

CTTCTTCAGTTGCCATTATTGATGTCAACGATTTACAAAAGGAATITTCACTGATTCTG 1320 $\begin{array}{llllllllllllllllllllll}S & S & S & V & A & I & I & D & V & N & D & L & Q & K & E & L & F & T & D & S\end{array}$

GTGCTGGTACTTTAATCAGAAGAGGTTACAGATTAATTAACAGAAACTCCTTGCGTGATT 1380 $\begin{array}{llllllllllllllllllll}G & A & G & T & L & I & R & R & G & Y & R & L & I & N & R & N & S & L & R & D\end{array}$

TTGGTAACCCAGACTTGTTAAGAAACGCATTGTTAAGAGACCCAGAAATCAAAACTGGTA 1440 $\begin{array}{llllllllllllllllllllll}F & G & N & P & D & \text { L } & \text { L } & \text { R } & \text { N } & \text { A } & \text { L } & \text { L } & \text { R } & \text { D } & \text { P } & \text { E } & \text { I } & \text { K } & \text { T } & G\end{array}$

AAGTGTCTGTTGCTTCATACTTGAAGTTITTGGATTCTGTTCAATTCAAGAGTTATGGAG 1500 $\begin{array}{llllllllllllllllllll}X & V & S & V & A & S & Y & L & K & F & L & D & S & V & Q & F & K & S & Y & G\end{array}$

ACGACCTITGGAAGTATTGGCTATTGTGGTAGAACAAAATGACAAAATTCCTAAATTAG 1560 D E P L E V L A

ACGAGTTTTTGTCATCCAAGACAGGTIGGTTAAACAATGTTACCGATAATATTTTCAATG 1620

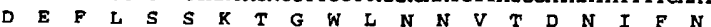

CTATCAAGAAAGATTACAGTCAATTATGTTGGGTTGTTATGAAAACGATGCCAACTTAC 1680 $\begin{array}{lllllllllllllllllllll}A & I & K & K & D & Y & S & Q & \text { L } & C & W & V & V & N & E & N & D & A & N & L\end{array}$

CTTGGTATTTCTCCAAATCAGATGGTTCATTTGCCAAGAATGGCCAAATCTMGTTTTGGT 1740

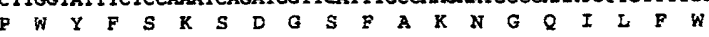

ACGGTTTAAACATTGACGAAGCTAGTAAATTGATTAAAGAATTTGATTCTTCATCTATRG 1800 $\begin{array}{llllllllllllllllllll}Y & G & L & N & I & D & E & A & S & K & \text { L } & I & K & E & P & D & S & S & S & I\end{array}$

ATCATCGTTGTCATCTTCTAAAGAATCTGGCGTATTCACATCTGCTCAACAAAAGCGTG 1860

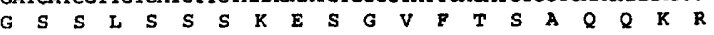

GTITCCACCACTCTACAGTCCGTAGAAACACCAATCCTAATCCTCCATTATCTGAAGGTA 1920 $\begin{array}{llllllllllllllllllll} & F & H & H & S & T & V & R & R & N & T & N & P & N & P & P & L & S & E & G\end{array}$

AGCAAACCGAGAGAAAAAAGTTGCTPPGATTGGTGCTAGAGGTTACACTGGACAAAACT 1980 $\begin{array}{llllllllllllllllllllll}K & Q & T & E & R & K & K & V & A & L & I & G & A & R & G & Y & T & G & Q & N\end{array}$

TGATTAAATTGATCGACAACCATCCATACTIGGATATTTCATATGTGTCGTCACGTGAAT 2040 $\begin{array}{lllllllllllllllllllll}\text { L } & I & K & \text { L } & \text { I } & \text { D } & \text { N } & \text { H } & \text { P } & \text { Y } & \text { L } & \text { D } & \text { I } & \text { S } & \text { Y } & \text { V } & \text { S } & \text { S } & R & \text { E }\end{array}$

TGGAAGGTCAAAAATTACAAGGTTACAACAAGATAACATTGTTTACTCCAACTTGCAAA 2100

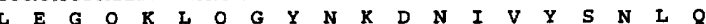

TTGAAGACATCAAGAGATTAGAAGAAAACAATGAGGTTGATGTTTGGGTTATGGCTTTAC 2160 $\begin{array}{llllllllllllllllllll}I & E & D & I & K & R & L & E & E & N & N & E & V & D & V & W & V & M & A & L\end{array}$

CTAATGGTGTTTGTAAACCATTTGTTGACACTATTGATTTAGTTCAGAACCCAAATTCTA 2220

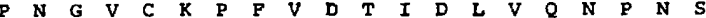

AAATTGTCGTTTATCTGCTGATTATCGTTTTGACACAACTGGTGAATGGACTTATGGAT 2280 $\begin{array}{llllllllllllllllllll}K & I & V & D & \text { L } & S & A & D & Y & R & \text { F } & \text { D } & \text { T } & \text { T } & G & \text { E } & \text { W } & T & Y & G\end{array}$

TACCAGAACTAAATGATCGTAAGACCATAGCTCAAGCTAAGAAAATTTCCAACCCAGGTT 2340 $\begin{array}{llllllllllllllllllll} & P & E & L & N & D & R & K & T & I & A & O & A & K & K & I & S & N & P & G\end{array}$

GCTATGCCACTGCTGCTCAAGTCGCCATTGCCCCGTTAAAAGAATATATTTCTGGAACTC 2400 $\begin{array}{llllllllllllllllllll}C & \mathbf{Y} & \mathbf{A} & \mathbf{T} & \mathbf{A} & \mathbf{A} & \mathbf{Q} & \boldsymbol{V} & \mathbf{A} & \mathbf{I} & \mathbf{A} & \mathbf{P} & \mathbf{L} & \mathbf{K} & \mathbf{E} & \mathbf{Y} & \mathbf{I} & \mathbf{S} & \mathbf{G} & \mathbf{T}\end{array}$

CAAGCATTTTTGGTGTTTCTGGCTATTCAGGTGCTGGAACCAAACATCTCCGAAGAACG 2460 $\begin{array}{lllllllllllllllllllll}P & S & I & F & G & V & S & G & Y & S & G & A & G & T & K & P & S & P & K & N\end{array}$

ACGTCAATTTATTGTCAAATAATTTAATTCCTTACTCGTTAACTGATCATGTACACGAAA 2520 $\begin{array}{lllllllllllllllllllll}D & V & N & L & L & S & N & N & L & I & P & Y & S & L & T & D & H & V & H & E\end{array}$

AAGAAATTAGTAGTCAATTAGGTTTACAAGTTGCATTTACCCCACACGTTGCTCAATGGT 2580 $\begin{array}{llllllllllllllllllll}K & E & I & S & S & Q & L & G & L & Q & V & A & F & T & P & H & V & A & Q & W\end{array}$

TTCAAGGTATTACTCATACTATCAACATTCCAATTAAAAAAGGATCTTTGACTTCTCGTG 2640

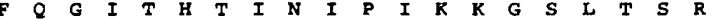

AAATTAGAAACATTTACCAAgATAGATATCAAGgTGAaAagCTAATCACCATTAGTGGag 2700 $\begin{array}{llllllllllllllllllll}E & I & R & N & I & Y & Q & D & R & Y & Q & G & E & K & L & I & T & I & S & G\end{array}$

AAGCACCACTTGTTAAAGACATTAGTGGCAAACACGGTGTCGTTGTTGGTGGCTPTGCTG 2760 $\begin{array}{lllllllllllllllllllll} & \text { A } & \text { P } & \text { L } & \text { V } & K & \text { C } & \text { D } & I & \text { S } & G & K & \text { H } & G & \text { V } & \text { V } & \text { V } & G & G & \text { P } & A\end{array}$

TCAACTCTAATGAAGACCGTGTTGTTATTGTTGCCACTATTGACAATTTGTTGAAAGGTG 2820 $\begin{array}{llllllllllllllllllll} & N & S & N & E & D & R & V & V & I & V & A & T & I & D & N & L & L & K & G\end{array}$

CAGCTACCCAATGTCTACAAAATATAAATTTGAGTCAAGAATTTGGTGAATACGATGGTA 2880 $\begin{array}{llllllllllllllllllllll}\text { A } & A & T & Q & C & L & Q & N & I & N & L & S & Q & E & F & G & E & Y & D & G\end{array}$

TTCCAACCGAATCCTTGATCAGAGGCTAAACTTGCCACAAAAAGAGTATGAAATTITITA 2940

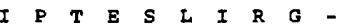

GATAGTTATTATITTAGTITIIITPCCTATAAGTTATATAAATAGACTITATATACAA 3000

TCATATACACTTGTTTGATTAGATTAAGTTTGATATTTCTCCCTITATATATACTTCT 3060 AATATITTATAGCCAATTA CEATCC

Fig. 2. Nucleotide sequence of a Scal-BamHI fragment encoding $C$. albicans ARG5,6 with its deduced amino acid translation. The presumed TATA box region (underlined) and the putative transcription termination signal (double underlined) are shown. Scal and BamHI sites are highlighted in bold.

pUC-ARG-U), a clone was selected which was shown by Southern blotting to integrate the URA3-blaster construction at the $A R G 5,6$ locus, yielding the heterozygous strain CNC40. Excision of URA3 was achieved on 5fluoroorotic acid plates, yielding strain CNC41. Strain CNC42 (homozygous arg5,6s strain) was obtained through a second round of transformation which allowed us to obtain arginine auxotrophs at a frequency of $15 \%$. Finally, excision of URA3 on 5-fluoroorotic

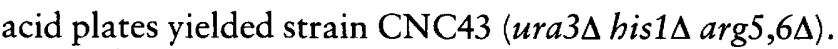
In an alternative approach, the C. albicans HIS1 gene (Pla et al., 1995) was used to replace an internal region of C. albicans ARG5,6. Strain CNC40 was transformed with pUC-ARG-H making use of HIS1 (see Methods) as a selectable marker (Fig. 1). Fifteen of $21 \mathrm{Ura}^{+} \mathrm{His}^{+}$ transformants $(70 \%)$ were shown to be arginine auxotrophs. This strain was efficiently transformed to arginine prototrophy with plasmid pAN8 (see Methods). 
The availability of an appropriate genetic marker, the $C$. albicans ARG5,6 gene, allowed us to check the feasibility of a single-step gene disruption in C. albicans. We

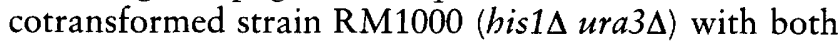
$U R A$ - and HIS-blaster constructions. A cotransformation frequency of approximately $10 \%$ was found, similar to that quoted by other authors (Kurtz et al., 1986, 1990). Ten per cent of the prototrophic clones were shown to be arginine auxotrophs (strain CNC44) carrying the correct (i.e. homologous) recombination event at the $A R G 5,6$ locus. This result is in general agreement with estimated frequencies for single recombination events $(70 \%$ and $15 \%)$.

We conclude that it is possible to generate a deletion in a specific $C$. albicans gene in a single transformation step. This should expedite the generation of homozygous null mutants by integrative transformation by reducing substantially the number of steps required to achieve the disruption of the two alleles at each genetic locus. Strains with different gene markers (like the ura3 $\Delta$ his $1 \Delta \arg 5,6 \Delta$ strain described in this work) and suitable for genetic transformation should allow the deletion of both alleles of a specific gene while maintaining the wild-type gene on an episomal plasmid.

\section{ACKNOWLEDGEMENTS}

We thank R. M. Pérez Díaz for her excellent technical assistance, F. Navarro-García for critical reading of the manuscript, M. L. Hernáez for helpful scientific comments and M. García Sáez for the expert sequencing technical work (Servicio de Secuenciación Automatizada de DNA de la Universidad Complutense). This investigation was supported by the grant FIS93/0183 from Fondo de Investigaciones Sanitarias. L. Monteoliva is the recipient of a fellowship from the Universidad Complutense de Madrid.

\section{REFERENCES}

Boonchird, C., Messenguy, F. \& Dubois, E. (1991). Characterization of the yeast $A R G 5,6$ gene: determination of the nucleotide sequence, analysis of the control region and of ARG5,6 transcript. Mol Gen Genet 226, 154-166.

Cannon, R. D., Jenkinson, H. F. \& Shepherd, M. G. (1990). Isolation and nucleotide sequence of an autonomously replicating sequence (ARS) element functional in Candida albicans and Saccharomyces cerevisiae. Mol Gen Genet 221, 210-218.

Cannon, R. D., Jenkinson, H. F. \& Shepherd, M. G. (1992). Cloning and expression of Candida albicans ADE2 and proteinase genes on a replicative plasmid in C. albicans and in Saccharomyces cerevisiae. Mol Gen Genet 235, 453-457.

Fonzi, W. A. \& Irwin, M. Y. (1993). Isogenic strain construction and gene mapping in Candida albicans. Genetics 134, 717-728.

Gil, C, Pomés, R. \& Nombela, C. (1988). A complementation analysis by parasexual recombination of Candida albicans morphological mutants. J Gen Microbiol 134, 1587-1595.

Gil, C., Pomés, R. \& Nombela, C. (1990). Isolation and characterization of Candida albicans morphological mutants derepressed for the formation of filamentous hypha-type structures. J Bacteriol 172, 2384-2391.

Gillum, A. M., Tsay, E. Y. H. \& Kirsch, D. R. (1984). Isolation of the
Candida albicans gene for orotidine- $5^{\prime}$-phosphate decarboxylase by complementation of $S$. cerevisiae ura3 and E. coli pyrF mutations. Mol Gen Genet 198, 179-182.

Gorman, J. A., Chan, W. \& Gorman, J. W. (1991). Repeated use of GAL1 for gene disruption in Candida albicans. Genetics 129, 19-24.

Goshorn, A. K. \& Scherer, S. (1989). Genetic analysis of prototrophic natural variants of Candida albicans. Genetics 123, 667-673.

Goshorn, A. K., Grindle, S. M. \& Scherer, S. (1992). Gene isolation by complementation in Candida albicans and applications to physical and genetic mapping. Infect Immun 60, 876-884.

Hanahan, D. (1988). Techniques for transformation of E. coli. In DNA Cloning, pp. 109-135. Edited by D. M. Glover. Oxford: IRL Press.

Herreros, E., García-Sáez, M. I., Nombela, C. \& Sánchez, M. (1992). A reorganized Candida albicans DNA sequence promoting homologous non-integrative genetic transformation. Mol Microbiol 6, 3567-3574.

Hill, J. E., Myers, A. M., Koerner, T. J. \& Tzagoloff, A. (1986). Yeast $/ E$. coli shuttle vectors with multiple unique restriction sites. Yeast 2, 163-167.

Hoyer, L. L., Magee, B. B., Rikkerink, E. H. \& Scherer, S. (1994). The ARG4 gene of Candida albicans. Gene 2, 213-218.

van Huffel, C., Dubois, E. \& Messenguy, F. (1992). Cloning and sequencing of $\arg 3$ and $\arg 11$ genes of Schizosaccharomyces pombe on a 10-kb DNA fragment. Heterologous expression and mitochondrial targeting of their translation products. Eur $J$ Biochem 205, 33-43.

Kelly, R., Miller, S. M., Kurtz, M. B. \& Kirsch, D. R. (1987). Directed mutagenesis in Candida albicans: one-step gene disruption to isolate ura3 mutants. Mol Cell Biol 7, 199-208.

Kurtz, M. B., Cortelyou, M. W. \& Kirsch, D. R. (1986). Integrative transformation of Candida albicans using a cloned Candida ADE2 gene. Mol Cell Biol 6, 142-149.

Kurtz, M. B., Cortelyou, M. W., Miller, S. M., Lai, M. \& Kirsch, D. R. (1987). Development of autonomously replicating plasmids for Candida albicans. Mol Cell Biol 7, 209-217.

Kurtz, M. B., Kelly, R. \& Kirsch, D. R. (1990). Molecular genetics of Candida albicans. In The Genetics of Candida, pp. 21-74. Edited by D. R. Kirsch, R. Kelly \& M. B. Kurtz. Boca Raton, FL: CRC Press.

Navarro-Garcla, F., Sánchez, M., Pla, J. \& Nombela, C. (1995). Functional characterization of the $M K C 1$ gene of Candida albicans, which encodes a mitogen-activated protein kinase homolog related to cell integrity. Mol Cell Biol 4, 2197-2206.

Odds, F. C. (1988). Candida and Candidosis, 2nd edn. London: Baillière Tindall.

Pearson, W. R. \& Lipman, D. J. (1988). Improved tools for biological sequence comparison. Proc Natl Acad Sci USA 85, 2444-2448.

Pereira, S. A. \& Livi, G. P. (1995). A GCN-like response in Candida albicans. Cell Biol Int 19, 65-69.

Pla, J., Pérez-Díaz, R. M., Navarro-García, F., Sánchez, M. \& Nombela, C. (1995). Cloning of Candida albicans HIS1 gene by direct homologous complementation of a histidine auxotroph using an improved double-ARS shuttle vector. Gene 165, 115-120.

Rosenbluh, A., Mevarech, M., Koltin, Y. \& Gorman, J. A. (1985). Isolation of genes from Candida albicans by complementation in Saccharomyces cerevisiae. Mol Gen Genet 200, 500-502.

Sadhu, C., Hoekstra, D., McEachern, M. J., Reed, S. I. \& Hicks, J. B. 
(1992). A G-protein alpha subunit from asexual Candida albicans functions in the mating signal transduction pathway of Saccharomyces cerevisiae and is regulated by the a1-alpha 2 repressor. Mol Cell Biol 12, 1977-1985.

Sambrook, J., Fritsch, E. F. \& Maniatis, T. (1989). Molecular Cloning: A Laboratory Manual. Cold Spring Harbor, NY: Cold Spring Harbor Laboratory.

Sanger, F., Nicklen, S. \& Coulson, A. R. (1977). DNA sequencing with chain-terminating inhibitors. Proc Natl Acad Sci USA 74, 5463-5467.

Scherer, S. \& Magee, P. T. (1990). Genetics of Candida albicans. Microbiol Rev 54, 226-241.

Received 1 July 1996; revised 27 September 1996; accepted 8 October 1996. 